wann dieses Gebiet in einem Jugendhilfe- oder einem eigenen Ausbildungshilfegesetz geregelt würde. ${ }^{162}$

Bei deren Ausgestaltung kam die Sozialabteilung den ländlichen Kommunen und den Ländern jetzt deutlich entgegen ${ }^{163}$ : Die Altersgrenze wurde auf 25 Jahre herabgesetzt, der Besuch einer Fach- oder Hochschule auf eine Soll-Leistung reduziert und der Besuch weiterführender Schulen an schärfere Voraussetzungen geknüpft. Nicht zuletzt wurde durch die generelle Verminderung der Einkommensgrenzen auch hier der Kreis möglicher Hilfeempfänger wieder stärker beschränkt. Das Hauptmonitum des Rechtsanspruchs auf diese Hilfen allerdings blieb bestehen. Die Begründung des Entwurfs versuchte die künftigen Belastungen der Träger zu bagatellisieren, denn im Falle eines Bundesgesetzes über Ausbildungshilfen würden diese Bestimmungen ,in ihrer Auswirkung erheblich an Bedeutung verlieren, vielleicht sogar ganz entfallen können". ${ }^{164}$

Doch die Hoffnung, mit dem weitgehenden Einlenken gegenüber dem Familienministerium den BSHG-Entwurf aus dem Schußfeld der Ressortkonflikte geholt und weitgehend kabinettsreif gemacht zu haben, trog: Tatsächlich beschäftigten die Auseinandersetzungen um das geplante Jugendhilfegesetz das Bundesinnenministerium auch noch das ganze Jahr 1959 hindurch.

\title{
3. Gesundheitliche Hilfen
}

Gemäß dem Ziel, eine „allgemeine Auffangstellung in den Wechselfällen des Lebens" $165 \mathrm{zu}$ schaffen, sah der erste Referentenentwurf für das BSHG relativ weitreichende Hilfen zur Aufrechterhaltung und Wiederherstellung der Gesundheit vor: neben den klassischen Aufgabenfeldern der Krankenhilfe und der Hilfe für werdende Mütter und Wöchnerinnen Maßnahmen der vorbeugenden Gesundheitshilfe und der Tuberkulosefürsorge. 166 Ähnlich wie bei den Hilfen für Jugendliche bewegte sich der Entwurf damit auf einem Terrain, das auch von anderen Leistungsträgern mit abgedeckt und für die eigene rechtliche Kodifikation beansprucht wurde. Krankenhilfe und Schwangeren- bzw. Wöchnerinnenhilfe waren bereits im bisherigen Fürsorgerecht als Pflichtleistungen verankert. ${ }^{167}$ Das auch in

162 Vgl. NDV 40 (1960), S. 178.

163 Vgl. SS 28-32 BSHG-Entwurf 3/1959, BAK, B 106/20646.

164 Begründung B zum BSHG-Entwurf 3/1959, S. 15, ADW, HGSt, SP-S XXV 1: 012-1/1.

165 Gerhard Scheffler, Neuordnung, S. 23.

$166 \mathrm{Zu}$ den Hilfen zur Pflege bzw. Hauspflege sowie zur medizinischen Rehabilitation für Behinderte siehe die beiden nachfolgenden Abschnitte.

167 Nach gängiger Rechtsauffassung, vgl. Muthesius, Grundlagen, S. 86; Jehle, Fürsorgerecht, S.143ff., umfaßte die Krankenhilfe alle nach dem jeweiligen wissenschaftlichen Kenntnisstand erforderlichen Behandlungsmaßnahmen einschließlich Krankenhauspflege und notwendiger Kuren. Viele Fürsorgeverbände schlossen entsprechende Verträge mit Ärzteorganisationen oder örtlichen Krankenkassen, die dann im Auftrag und auf Kosten der Fürsorgeverbände tätig wurden; vgl. NDV 35 (1955), S.70ff. Für die Wochenhilfe sah $₫ 12$ RGr. ggf. ärztliche Behandlung, einen Entbindungskostenbeitrag und Wochen- sowie Stillgeld vor; diese Hilfen sollten den Leistungen der gesetzlichen Krankenversicherung entsprechen. Gemäß $\ 6$ Abs. 3 RFV schrieben die Fürsorgeverbände 
den fünfziger Jahren noch zentrale und vordringliche Aufgabengebiet der TbcFürsorge sollte zunächst analog zum KBG in einem Sondergesetz geregelt und später in das Bundessozialhilfegesetz eingearbeitet werden. Mit der „vorbeugenden Gesundheitshilfe" hingegen versuchte die Sozialabteilung, zumindest für die Gruppe der künftigen Sozialhilfeempfänger medizinische Leistungen zu ermöglichen, die auf der Höhe der Zeit standen, und damit die nach zeitgenössischer Expertenmeinung prekäre Situation des öffentlichen Gesundheitswesens wie auch Defizite der gesetzlichen Krankenversicherung wenigstens für einen kleinen Teil der Bevölkerung zu beheben.

\section{Vorbengende Gesundheitsfürsorge, Krankenhilfe, Hilfe für Schwangere und Wöchnerinnen}

In den zwanziger Jahren hatte mit der Sozialhygiene die Gesundheitsfürsorge als traditionelle Domäne der Kommunen einen deutlichen Aufschwung genommen und dabei wegen mangelnder Therapiemöglichkeiten die Prophylaxe zunehmend an Bedeutung gewonnen: Gesundheitsfürsorge für Säuglinge, Klein- und Schulkinder, Jugendliche und Mütter ebenso wie die Prävention gefährlicher Infektionskrankheiten jenseits seuchenpolizeilicher Schutzmaßnahmen wurden zumindest in entsprechend ausgestatteten großstädtischen Gesundheitsämtern ausgebaut mit Säuglingsfürsorgestellen, Schulärzten, Schulzahnärzten, Röntgenuntersuchungen zur Tuberkuloseabwehr, Maßnahmen der Frühdiagnose für Geschlechtskranke etc. Allerdings richtete sich auch diese Arbeit immer auf das individuelle Verhalten der Betroffenen; für eine umfassende, auch soziale Notlagen bearbeitende und reichsweit einheitliche öffentliche Gesundheitsfürsorge fehlten in der Weimarer Republik die materiellen Ressourcen. ${ }^{168}$

Mit dem „Gesetz zur Vereinheitlichung des Gesundheitswesens“ (GVG) war 1934/35 das öffentliche Gesundheitswesen verstaatlicht und ein reichsweites Netz von Gesundheitsämtern geschaffen worden, die neben Aufgaben der Seuchenpolizei, Umwelthygiene und - sofern nicht von der NSV übernommen - Gesundheitsfürsorge nun vor allem die nationalsozialistische Bevölkerungs- und Rassenpolitik umzusetzen hatten. ${ }^{169}$ In unserem Zusammenhang interessiert, daß das Gesetz und eine Verordnung vom März 1935 zwar die Aufgaben der öffentlichen Gesundheitsfürsorge ausdrücklich auflisteten, etwa für den wichtigen Teilbereich der Mütterfürsorge aber weder deren Umfang noch Trägerschaft regelten. ${ }^{170}$

Nach 1945 war die Entwicklung des öffentlichen Gesundheitswesens sehr unterschiedlich: Ein Teil der Gesundheitsämter wurde wieder kommunalisiert, ein

örtlich für die Wochenfürsorge eine besondere Einkommensgrenze vor, die Mitte der fünfziger Jahre meist beim doppelten Richtsatz lag.

$168 \mathrm{Vgl}$. ausführlich Sachße/Tennstedt, Geschichte, Bd.2, S.114ff.; ferner die Literaturhinweise zur Geschichte der Gesundheitsfürsorge bis 1945 bei Andreas Wollasch, Tendenzen, S. 11, 18.

169 Vgl. ausführlich Sachße/Tennstedt, Geschichte, Bd.3, S. 97ff., 166ff.; Süß, Gesundheitspolitik, passim, sowie Vossen, Gesundheitsämter.

170 Vgl. $\int 3$ GVG, RGBl. I S. 531, und \$S 55-61 der Dritten DVO zum GVG vom 30.3. 1935, RGBl. I S. 327. 
Teil blieb staatlich, das heißt Teil der Länderverwaltung; der bundesrechtliche Status des (etwas bereinigten) GVG und die Gesetzgebungskompetenz des Bundes waren umstritten, so daß auch die Leistungen in den Ländern und Regionen oft stark voneinander abwichen. ${ }^{171}$ Nach der Bewältigung der drängendsten Gesundheitsprobleme der Nachkriegszeit gerieten zunehmend die neuen und besseren Möglichkeiten der Vorbeugung und Früherkennung in den Blick ebenso wie der im internationalen Vergleich immer noch ungünstige Gesundheitszustand der bundesdeutschen Bevölkerung mit einer überdurchschnittlich hohen Säuglingssterblichkeit. ${ }^{172}$ Es mehrten sich daher die Stimmen, die wie Abteilungsleiter Kitz einen Ausbau der „vorbeugenden Gesundheitsfürsorge“ forderten, die im Rahmen der gesetzlichen Krankenversicherung bislang nur ungenügend geleistet wurde. ${ }^{173}$

Über die notwendigen Maßnahmen herrschte dabei unter Gesundheitsexperten weitgehend Einigkeit: Verstärkung des Mutterschutzes auch für nicht erwerbstätige Frauen, regelmäßige Vorsorgeuntersuchungen für werdende Mütter, Säuglinge, die bisher kaum erfaßten Kleinkinder, für Schulkinder und Jugendliche, Impfschutz, zahnmedizinische Prophylaxe, Ausbau der Erholungsfürsorge, gesundheitliche Aufklärung und Erziehung, Einrichtung von Beratungsstellen, Ausbau der Wohnungsfürsorge u.a.m. ${ }^{174}$ Massive Divergenzen dagegen bestanden hinsichtlich der organisatorischen, rechtlichen und damit auch finanziellen Wege, um diese Maßnahmen großflächig umzusetzen. ${ }^{175}$ Da bereits rund drei Viertel der Bevölkerung durch die gesetzliche Krankenversicherung erfaßt wurden, sprach viel für Organisationsformen, in denen Sozialversicherung und niedergelassene Kassenärzte die führende Rolle spielten. Umgekehrt blieb das Problem der in der ambulanten Medizin arztrechtlich nicht gestatteten „zu- bzw. nachgehenden“ Fürsorge, zumal im Rahmen eines Konzepts gruppenbezogener Prävention, das nur über öffentliche Organisation lösbar schien. Hier trafen standes- und organisationspolitische Interessen von niedergelassenen Ärzten und Krankenkassen auf Kompetenzansprüche der Gesundheitsämter und Amtsärzte, die wiederum mit den Kommunalisierungsbestrebungen der Städte und Kreise in Konflikt gerieten. Schon als Wilhelm Hagen von der Gesundheitsabteilung des Bundesinnenministeriums 1953 die Initiative für eine bundeseinheitliche Neuregelung des öffentlichen

171 Vgl. Rainer, Gesundheitsfürsorge, S. 252ff.; Labisch, Entwicklungslinien, S.753; Kühn, Jugendamt, S. 62ff.; Wasem u.a., Gesundheitswesen, S. 485ff.

172 Vgl. Hagen, Gesundheitsfürsorge, S. 240ff.; ders., Jugendgesundheit, S. 152ff.

173 Vgl. Kitz, Gegenwartsfragen, S. 333.

174 Vgl. NDV 30 (1950), S.3, 186f.; Muthesius, Die kommunale Fürsorge, S. 253; Entschließung des DST von 1951, NDV 31 (1951), S.250f.; Grundlagen des sozialen Gesamtplanes der SPD vom 14.9.1952, in: Richter, Sozialreform, Bd.6 G II, S. 2; Vorschläge des CDU-Bundesausschusses für Sozialpolitik vom April 1953, in: ebenda, G I, S. 7; Hagen, Gesundheitsfürsorge, S.240ff.; nur zum Teil in den sog. „Unkeler Beschlüssen“ des „Ausschusses für Krankheitsbekämpfung (einschließlich Krankenversicherung)“ des BMA-Beirats vom 3./4.10.1955, BABl. 1955, S.1038f.; Entschließung des CDUBundesparteitags vom 26.-29.4.1956, in: Richter, Sozialreform, Bd.6 G I, S. 17; Sozialplan, S. 32ff.; ferner die Nachweise bei Labisch/Tennstedt, Prävention, S.141ff.

175 Vgl. insgesamt Manger-König, Gesundheitsdienst; Labisch/Tennstedt, Prävention, S. $136 \mathrm{ff}$. 
Gesundheitsdienstes ergriffen und einen Gesetzentwurf über die vorbeugende Gesundheitsfürsorge als dessen Aufgabe vorbereitet hatte, war das ohne nachhaltige Resonanz geblieben. ${ }^{176}$

Im Fürsorgerecht galt bislang nur die allgemeine und unverbindliche Möglichkeit zu vorbeugender Fürsorge des $\mathbb{3}$ RGr., die Polligkeit schon in seinem Reformgutachten von 1946 für ungenügend erachtet hatte ${ }^{177}$ und der 1950 zumindest für Empfänger von Kriegsfolgenhilfe mehr gesundheitsfürsorgerische Durchschlagkraft verliehen worden war. ${ }^{178}$ Wie die Erziehungshilfen hatte Gottschick in seinen ersten Konzepten das schwierig abzugrenzende Gebiet der vorbeugenden Gesundheitsfürsorge noch ausgeklammert, dann auf Anregung der kommunalen Sachverständigen aber zumindest vorbeugende Erholungsmaßnahmen als KannLeistung vorgesehen. ${ }^{179}$ Das war Scheffler offensichtlich zu wenig 180 : Nachdem zuvor die DST-Hauptversammlung im Juni 1956 ihre Bereitschaft zu breit angelegter, natürlich auf der Basis kommunaler Gesundheitsämter operierender vorbeugender Gesundheitsfürsorge verkündet hatte, sah der BSHG-Entwurf vom November 1956 entsprechende Maßnahmen als Pflichtleistung auch der Sozialhilfe vor. ${ }^{181}$ Dabei ging es Scheffler nicht um eine Beeinträchtigung der allgemeinen Aufgaben der Gesundheitsämter, sondern um die Sicherung der notwendigen Maßnahmen im Einzelfall, eine Auffassung, die aus unterschiedlichen Motiven ebenso von den Länderreferenten wie indirekt vom SPD-Sozialplan von 1957 gestützt wurde. ${ }^{182}$

Dem eng mit der Sozialabteilung zusammenarbeitenden Theodor Marx allerdings gingen diese Bestimmungen nicht weit genug. Er war der Ansicht, daß ein umfassendes Bundesgesundheitsgesetz im Sinne von Hagen zwar dringend not-

176 Tatsächlich verlor die Gesundheitsfürsorge des öffentlichen Gesundheitsdienstes immer mehr an Bedeutung infolge des Ausbaus der Leistungen der gesetzlichen Krankenversicherung und der Kompetenzerweiterung der niedergelassenen Ärzte; vgl. Labisch, Entwicklungslinien, S. 753f.; Labisch/Tennstedt, Prävention, S. 149ff.; Kühn, Jugendamt, S. 72f., 82f. Das seit der Therapierbarkeit nicht mehr umfassende allgemeine Fürsorgemaßnahmen erfordernde Sondergebiet der Geschlechtskrankheiten war 1953 gesetzlich geregelt worden. Die dabei vorgesehenen Arbeitsgemeinschaften von öffentlichem Gesundheitsdienst, Versicherungen und niedergelassenen Ärzten wie auch die Meldepflicht funktionierten aus Sicht der Gesundheitsämter allerdings nicht befriedigend; vgl. Hagen, Gesundheitsfürsorge, S. 244.

177 Vgl. Rundschreiben 1 (1946), S. 51.

178 Laut $\int 10$ des Ersten Überleitungsgesetzes vom 28.11.1950 galt auch vorbeugende Erholungsfürsorge für Mütter, Kinder und Jugendliche als verrechnungsfähig im Sinne einer Pflichtleistung der Fürsorge, BGBl. S. 773.

179 Vgl. $\ 36$ des Teilentwurfs vom 25.5.1956, BAK, B 106/20648; Vermerk über die Besprechung mit den Sachverständigen am 3.5.1956, BAK, B 106/9789/2.

180 So eine Randglosse Schefflers zu $\mathbb{3} 36$ des Teilentwurfs vom 25.5.1956, BAK, B $106 / 20648$.

181 Vgl. \S 59-61 BSHG-Entwurf vom 24.11.1956, ebenda; Der Städtetag 9 (1956), S.355ff. Zudem gab der Wechsel Hagens, der die Einbeziehung solcher Hilfen in das Fürsorgerecht ablehnte, vgl. Vermerk Referat V A 4 vom 18.7.1957, BAK, B 106/9786/2, an die Spitze des Bundesgesundheitsamtes zum 1.7.1956 der Sozialabteilung freiere Hand.

182 Vgl. Abteilungsleitervorlage vom 16.11.1956, BAK, B 106/20652; Besprechung mit den Referenten der Länder am 5./6.2.1957, BAK, B 106/9789/2; Sozialplan, S.32, 64ff., $125 \mathrm{ff}$. 
wendig, aber angesichts der unklaren Legislativkompetenz des Bundes in absehbarer Zeit kaum zu erwarten war. Daher drängte er auf eine möglichst weitgehende, dann auch über die fürsorgerische Hilfsbedürftigkeit hinausgehende vorläufige Verankerung der vorbeugenden Gesundheitsfürsorge, insbesondere einer bestimmten Zahl von Vorsorgeuntersuchungen, im Fürsorgegesetz, um dadurch bundesweit das Niveau der öffentlichen Gesundheitsfürsorge zu heben und zu vereinheitlichen. ${ }^{183}$ Möglicherweise verfolgte er darüber hinaus das Ziel, Aufgaben des allgemeinen Gesundheitswesens zu Maßnahmen der öffentlichen Fürsorge gemäß Art.74 GG zu erklären und so quasi durch die Hintertür eine Gesetzgebungskompetenz des Bundes begründen zu können. ${ }^{184}$ Diese Strategie versuchte er innerhalb des DST, des DV und des Beirats durchzusetzen - ohne Erfolg. ${ }^{185}$ Zwar war man im Innenministerium keineswegs bereit, wie von Marx' internen Gegenspielern im DST-Gesundheitsausschuß gewünscht, ganz auf die vorbeugende Gesundheitsfürsorge zu verzichten, was zudem die Gefahr einer vorübergehenden Gesetzeslücke barg. Die Gesundheitspolitiker im DST und DV fürchteten nämlich, daß eine Übergangslösung im Fürsorgerecht das gewünschte Bundesgesundheitsgesetz verzögere und präjudiziere, daß dann die Gesundheitsfürsorge generell den Gesundheitsämtern genommen und deren Stellung gegenüber den Sozialämtern dauernd geschwächt werde. ${ }^{186}$ Doch bereits Ende April 1957 erklärte Scheffler intern, auf dem Fürsorgetag 1957 dann öffentlich, daß für die Gesundheitsfürsorge für die Gesamtbevölkerung nicht die subsidiäre Fürsorge zuständig sei; allgemeine Vorsorgeuntersuchungen etwa, ohnehin kaum mit Einkommensgrenzen zu vereinbaren, seien nicht Aufgabe der Sozialhilfe, sondern Voraussetzung für deren Tätigwerden im Einzelfall. ${ }^{187}$ Offensichtlich wollte Scheffler das künftige BSHG nicht mit allen offenen Posten der Sozialreform überfrachten.

Tatsächlich hätte die Sozialabteilung auch im eigenen Hause eine solche Linie kaum durchsetzen können: Die Gesundheitsabteilung erhob ohnehin starke Bedenken gegen den Einbau des KBG und des künftigen THG, da damit auch allgemeine gesundheitsrechtliche Regelungen in das Sozialhilfegesetz übernommen werden müßten. ${ }^{188}$ Der Fürsorgetag im November stützte das Vorgehen des

183 Vgl. Niederschriften über die Sitzungen des Arbeitsausschusses für Fragen der Fürsorge am 30.11./1.12. 1956 und 3./4.5.1957, ADW, HGSt 6769; Vermerk Referat V A 4 vom 16. 4.1957, BAK, B 106/9786/2; Niederschrift über die Sitzung des DST-Arbeitskreises „Fürsorgerecht“ am 7./8.5.1957, von Marx formulierte Thesenvorschläge zur Neuordnung der Krankheitsbekämpfung vom 10.5.1957, Rainer an Marx am 13.9.1957, Abschrift, LAB, B Rep. 142-9, 1282.

184 Vgl. Scheffler im Arbeitsausschuß für Fragen der Fürsorge am 3./4. 5.1957, Niederschrift, ADW, HGSt 6769.

185 Vgl. die Niederschriften über die Sitzungen des Arbeitsausschusses am 14./15. 6. 1957, ebenda, sowie des DST-Sozialausschusses am 26./27.9.1957, Auszug, LAB, B Rep. 142-9, 1282.

186 Vgl. Niederschrift über die Sitzung des DST-Gesundheitsausschusses am 24./25. 5. 1957, Auszug; Rainer an Marx am 13.9.1957, Abschrift, LAB, B Rep. 142-9, 1282; Vermerk Referat V A 4 vom 18.7.1957, BAK, B 106/9786/2.

187 Vgl. Vermerke Referat V A 4 über Besprechungen mit Abteilungsleiter am 29./30. 4. und 5.7.1957, BAK, B 106/9789/2; Gerhard Scheffler, Neuordnung, S. 30f.

188 Vgl. Vermerke Referat V A 4 vom 18.2. und 5.3.1958, BAK, B 106/20643. 
Innenministeriums und wünschte keine allgemeinen gesundheitsrechtlichen Bestimmungen für das BSHG; vielmehr forderte die Arbeitsgruppe E, gleichzeitig mit dem Bundessozialhilfegesetz und einem Bundesjugendhilfegesetz ein Bundesgesundheitsgesetz vorzulegen, nötigenfalls durch eine Änderung des Grundgesetzes. Anders als im Falle der Jugendhilfe konnte diese Junktimsforderung die Zeitpläne der Sozialabteilung allerdings - von der unklaren Rechtslage einmal abgesehen - kaum gefährden, denn das Gesundheitswesen ressortierte im eigenen Ministerium und es fehlte hier der entscheidende Verknüpfungspunkt des Konflikts um die freie Wohlfahrtspflege. Allerdings übernahm auch die Arbeitsgruppe E den von Marx und dem DV-Gesundheitsausschuß mit Hagen ausgehandelten Kompromiß: Ein präziser Aufgabenkatalog sollte zumindest für die künftigen Fürsorgeempfänger die modernsten Mindestleistungen per Rechtsanspruch sichern und gleichzeitig eine Vorreiterrolle für das erhoffte Gesundheitsgesetz übernehmen. ${ }^{189}$

Zunächst unterstützte auch der Fürsorgeausschuß des Beirats diese Forderungen, geriet damit aber in Gegensatz zu dem Beirats-Ausschuß für Krankheitsbekämpfung, der erklärte, daß den künftigen Sozialhilfeempfängern nicht mehr als den gesetzlich Krankenversicherten gewährt werden dürfe, die schließlich Beiträge geleistet hätten. ${ }^{190} \mathrm{Zu}$ den Pflichtleistungen der Krankenkassen gehörten regelmäßige Vorsorgeuntersuchungen aber nicht. Nach anfänglichem Widerstand lenkte der Fürsorgeausschuß in diesem Punkt ein und verzichtete auf die Festschreibung einer bestimmten Zahl von Vorsorgeuntersuchungen; mit der Forderung nach weiterhin zeitlich unbegrenzter Krankenhilfe und gleichwertigen Leistungen auch für Familienmitglieder allerdings war er über den Limits der Krankenversicherung geblieben. ${ }^{191}$

Auch der Referentenentwurf vom Juli 1958 sah zwar die „ärztliche Untersuchung zur möglichst frühzeitigen Erkennung einer gesundheitlichen Gefährdung“ vor, benannte diese aber nicht ausdrücklich in zeitgenössischer Terminologie als "Vorsichtsuntersuchung" und beließ deren Anwendung im behördlichen Ermessen. ${ }^{192}$ Besonders deutlich offenbarte sich damit das schon von Muthesius beschriebene Dilemma einer modernen Sozialhilfe, die einerseits notwendige Leistungen auch und gerade präventiv erbringen, andererseits aber die postulierte Vorleistungspflicht anderer Träger wahren wollte. Der Referentenentwurf ging ohnehin über das Nachrangprinzip hinaus, indem er auf „vorbeugende Gesundheitshilfe" einen Rechtsanspruch einräumte und einen Maßnahmenkatalog von der Kindererholung bis zur Müttergenesung aufführte. Diese Bestimmungen wurden von der Gesundheitsabteilung schließlich toleriert. ${ }^{193}$

189 Vgl. Neuordnung des Fürsorgerechts, S. 228ff., 266, 269, 434f.; Rainer, Gesundheitsfürsorge.

190 Vgl. die Niederschriften über die Sitzungen des Arbeitsausschusses für Fragen der Fürsorge am 30./31.1. und 6./7.6.1958, letztere mit Anlage 5, ADW, HGSt 6769.

191 Vgl. NDV 38 (1958), S. $306 f . ; 39$ (1959), S. $132 \mathrm{ff}$.

192 \$51 des BSHG-Entwurfs 7/1958, BAK, B 106/20643.

193 Vgl. Vermerk Referat V A 4 vom 28.5.1958 sowie Abteilung IV an Abteilung V am 11.6. 1958; \51 Abs. 4 BSHG-Entwurf 7/1958, ebenda. 
Auf Krankenhilfe und die Hilfe für Schwangere und Wöchnerinnen bestand wie im bisherigen Recht ein Rechtsanspruch (für den erweiterten Personenkreis innerhalb der allgemeinen Einkommensgrenzen für die Hilfe in besonderen Lebenslagen), die einzelnen materiellen Leistungen wurden in Anlehnung an bereits übliche Rechtsauslegung präzisiert bzw. etwas erweitert. ${ }^{194}$ Die Leistungen sollten mindestens den Pflichtleistungen der Krankenkassen entsprechen. Neben dem sozialreformerischen Ziel einer Leistungsangleichung der Sozialleistungsträger hatte diese Regelung auch praktische Gründe, boten doch Reichsversicherungsordnung und Satzungen der Ortskrankenkassen den örtlichen Sozialämtern eine hilfreiche Orientierung für die eigene Leistungsgewährung. ${ }^{195}$ Darüber hinaus garantierte die Bindung an die Krankenversicherung einen bundesweit einheitlichen Mindeststandard der gesundheitlichen Hilfen der künftigen Sozialhilfe. Wie sich allerdings bald zeigen sollte, barg gerade diese Relation zur Krankenversicherung erhebliches Konfliktpotential. Insgesamt hatte der Entwurf somit zwar nicht alle Details der Forderungen der Fürsorgereform-Gremien erfüllt, aber die bisher weitgehend dem behördlichen Ermessen überlassenen gesundheitlichen Fürsorgeleistungen durch Konkretisierung verrechtlicht und durch den Rechtsanspruch auf präzise vorbeugende Hilfen deutlich ausgebaut.

Bis zu ihrer endgültigen Regelung im BSHG wurden diese Vorschriften jedoch zum Teil erheblich geändert: Besonders einschneidend war, daß der Rechtsanspruch auf „vorbeugende Gesundheitshilfe“ schon im zweiten Referentenentwurf auf eine Soll-Verpflichtung des Sozialhilfeträgers vermindert wurde. ${ }^{196}$ Hier hatte abermals die Koalition aus Bundesfinanzministerium, der Mehrzahl der Länderreferenten und der ländlichen kommunalen Spitzenverbände ihre Effektivität bewiesen. Ein Rechtsanspruch sei nicht praktikabel - etwa im Falle widersprüchlicher ärztlicher Gutachten - bzw. nicht finanzierbar; denn das geltende Versicherungs- und Versorgungsrecht sah solche Hilfen fast nur als Kann-Leistungen vor, so daß Nichtversicherte gegenüber Beitragszahlern besser gestellt oder die Sozialhilfeträger u.U. auch für minderbemittelte Krankenversicherte vorsorgepflichtig würden. ${ }^{197}$ Auf Betreiben seines Finanzausschusses forderte der Bundesrat sogar die Abschwächung zur Kann-Leistung ${ }^{198}$, was aber von der Bundesregierung zurückgewiesen wurde, zumal zumindest jenseits der Partikularperspektive des unmittelbar betroffenen Trägers auch die volkswirtschaftliche Kostenersparnis durch medizinische Prävention ins Feld geführt werden konnte und ohnehin nur

194 Vgl. SS 52, 54 BSHG-Entwurf 7/1958, ebenda; neu war etwa die Verpflichtung, ggf. auch eine (von Ärzten propagierte) Anstaltsentbindung zu finanzieren.

195 Vgl. Besprechung mit den Fürsorgereferenten der Länder am 5./6.2.1957, BAK, B 106/9789/2.

196 Vgl. $₫ 33$ des BSHG-Entwurfs 3/1959, BAK, B 106/20646.

197 Vgl. Niederschrift über die Besprechung mit den Vertretern der obersten Landessozialbehörden am 21./22.10.1958, LAS Abt. 761 Nr. 8874; Stellungnahmen der bayerischen kommunalen Spitzenverbände vom 14.10.1958, des DLT vom 17.11.1958 und des DGT vom 1.12. 1958 sowie Vermerk Referat V A 4 vom 11.12.1958, BAK, B 106/9686.

198 Vgl. die Änderungsempfehlungen des Bundesratsausschusses für Innere Angelegenheiten zum BSHG-Entwurf vom 11.3.1960, S.15, BR, Drucksachen 1960, Drs. 53/1/60; Sitzung des Bundesrates am 18.3.1960, S.340, BR 1960, Sten. Ber. 
der Personenkreis innerhalb der allgemeinen Einkommensgrenze in Frage kam. ${ }^{199}$ Auch der kommunalpolitische Bundestagsausschuß blieb bei der Soll-Bestimmung, hätte doch eine Kann-Vorschrift sogar eine Verschlechterung gegenüber dem bisherigen Rechtszustand bedeutet. Als Soll-Bestimmung wurde dann die vorbeugende Gesundheitshilfe Gesetz.

Darüber hinaus verzichtete der zweite Referentenentwurf wie auch bei anderen Hilfen im Interesse der Kommunen auf den beispielhaften Aufgabenkatalog und erwähnte neben allgemeinen Erholungsmaßnahmen und Vorsorgekuren nur noch die „Müttererholung in geeigneten Müttergenesungsheimen“200 - letzteres ebenso ein Zugeständnis an eine zeitgenössische Notwendigkeit wie an den Bundespräsidenten, der im Interesse des von seiner Ehefrau initiierten Deutschen Mütter-Genesungswerks im November 1958 indirekt interveniert hatte. ${ }^{201}$ Die ursprünglich von Marx so vehement geforderten allgemeinen Vorsorgeuntersuchungen enthielt der Entwurf bereits nicht mehr. Erst der Bundesrat veranlaßte die Einfügung einer Kann-Bestimmung für Vorsorgeuntersuchungen zur Früherkennung, um vor allem den Entwicklungen der Krebs-Diagnostik Rechnung zu tragen. ${ }^{202}$ Somit hatten die ursprünglich weitreichenden Präventionspläne nur beschränkt Eingang in das BSHG gefunden. Und dies, obwohl - besser: gerade weil - weder die Pläne für ein Bundesgesundheitsgesetz noch für eine stärkere Verankerung der Prävention in der gesetzlichen Krankenversicherung weiter gediehen waren.

Tatsächlich erwies sich die Bindung an die Krankenversicherung schließlich als Hemmschuh für die fürsorgerischen Reformpläne. Die Absicht, dadurch einen bundesweit einheitlichen Mindeststandard zu etablieren, blieb solange obsolet, als die Pflichtleistungen der Krankenkassen hinter den geplanten Sozialhilfen zurückblieben. In der von Adenauer bereits 1957 angekündigten grundlegenden Reform der Krankenversicherung waren zwar deutliche Leistungsverbesserungen auch zur Prophylaxe vorgesehen, die durch eine Umstellung der ärztlichen Honorierung und durch eine Selbstbeteiligung der Patienten an den Kosten unter Kontrolle gehalten werden sollten. ${ }^{203}$ So sah ein Anfang Januar 1960 dem Bundestag vorgelegter Regierungsentwurf u.a. einen Anspruch auf einige Vorsorgeunter-

199 Vgl. die Auffassung der Bundesregierung zu der Stellungnahme des Bundesrates, S. 83, BT, 3. Wp. 1957, Anlagen, Bd.67, Drs. 1799, Anlage 3.

200 Vgl. DST-Stellungnahme vom 27.11.1958, BAK, B 106/9686; Niederschrift der Sitzung des DLT-Sozialausschusses am 2./3.7.1959, BAK, B 172/444-02/2, Beiheft; $\mathbb{S} 33$ des BSHG-Entwurfs 3/1959, BAK, B 106/20646.

201 Die Geschäftsführerin des Mütter-Genesungswerks Antonie Nopitsch hatte in einem Brief an Heuss eine mangelnde Berücksichtigung der Müttergenesungsfürsorge im BSHG-Entwurf kritisiert und war kurz darauf vom Bundespräsidenten persönlich empfangen worden. Ihrem Wunsch entsprechend sicherte Gottschick durch die neue Formulierung, daß diese Maßnahmen praktisch nur in Heimen des Mütter-Genesungswerks durchgeführt würden; vgl. Nopitsch an Bundespräsident am 12.11.1958 (Durchschlag), sowie Vermerk Referat V A 4 vom 14.11.1958, BAK, B 106/9686; Gottschick an die CDU-Bundestagsabgeordnete Welter am 10.6.1959, BAK, B 106/20644.

202 Vgl. \36 Abs. 1 BSHG.

$203 \mathrm{Zu}$ den Plänen für eine Neuordnung der Krankenversicherung und ihrem Scheitern vgl. ausführlich Reucher, Reformen. 
suchungen (auch während der Schwangerschaft) und auf Krankenhauspflege sowie Leistungsverbesserungen für mitversicherte Familienangehörige vor. ${ }^{204}$ Doch die heftig umkämpfte Reform scheiterte Anfang 1961 schließlich auch im Bundestag.

Das wirkte sich vor allem auf die Schwangerenhilfe der Sozialhilfe aus. Auf Drängen des DST, des DV und der Spitzenverbände der freien Wohlfahrtspflege und angesichts von Presseberichten über die hohe Müttersterblichkeit in der Bundesrepublik hatte die Sozialabteilung in den zweiten Referentenentwurf nämlich doch Schwangerschaftsuntersuchungen in den Katalog der Pflichtleistungen eingefügt. ${ }^{205}$ Doch mit dem Scheitern der Krankenversicherungsreform mußte diese Vorschrift wieder entfallen, wollte man auf die in der Verwaltung bereits eingespielte Leistungsgewährung anhand der Reichsversicherungsordnung nicht wieder verzichten. Wie bereits im Mai 1959 von den Wohlfahrtsministern der Länder befürchtet, wirkte sich hier die Bindung an die Krankenkassenleistung also vorläufig als Leistungsdeckelung aus. ${ }^{206}$

Die ausdrückliche Anknüpfung auch der Krankenhilfe an die gesetzliche Krankenversicherung war nach ganz anders begründeten Protesten bereits im zweiten Referentenentwurf wieder entfallen. Während der DLT die Koppelung an externe Mindestmaßstäbe als „allgemeine Aufweichung bisher bestehender Leistungsgrenzen“ kritisierte ${ }^{207}$, fürchteten Ortskrankenkassen wie Kassenärzte den umgekehrten Effekt: Hohe, mit einem Rechtsanspruch versehene Leistungen der Sozialhilfe würden den Wert der beitragspflichtigen gesetzlichen Krankenversicherung weitgehend illusorisch machen, eine Art staatlicher Gesundheitsdienst werde die genossenschaftliche Selbsthilfeeinrichtung Krankenversicherung allmählich aufsaugen. ${ }^{208}$ Da Bundesarbeits- und Bundeswirtschaftsministerium diese Forderungen unterstützten, verzichtete das Innenministerium auf den Passus, was sich nach dem Scheitern der Neuordnungspläne für die Krankenversicherung im Sinne des Hilfsbedürftigen letztlich als Vorteil erwies. ${ }^{209}$ Eine umfassende Definition der Krankenhilfe im BSHG ermöglichte individuelle Hilfe auch oberhalb der

204 Vgl. $\$ \int 117 f f ., 207 f ., 216 f f$. des Entwurfs eines Gesetzes zur Neuregelung des Rechts der gesetzlichen Krankenversicherung, BT, 3. Wp. 1957, Anlagen, Bd. 65, Drs. 1540.

205 Vgl. $\$ 35$ des BSHG-Entwurfs 3/1959, BAK, B 106/20646, sowie $\ 36$ des BSHG-Regierungsentwurfs, Februar 1960, BT, 3. Wp. 1957, Anlagen, Bd.67, Drs. 1799.

206 Vgl. Niederschrift über die Konferenz der für das Wohlfahrtswesen zuständigen Minister der Länder am 22.5.1959, BAK, B 106/20644. In der Hoffnung auf künftige Änderungen des Krankenversicherungsrechts wurde statt dessen auf Veranlassung des kommunalpolitischen Bundestagsausschusses ein - noch bedeutungsloser - Passus eingefügt, wonach die Hilfe für werdende Mütter auch Vorsorgeuntersuchungen umfasse, „soweit diese nach den Vorschriften über die gesetzliche Krankenversicherung zu gewähren sind“, $\ 38$ BSHG; dazu Gottschick, Bundessozialhilfegesetz, 1962, S. 161.

207 Stellungnahme des DLT vom 17.11.1958, BAK, B 106/9686.

208 Vgl. Vermerk Referat V A 4 vom 18.7.1957, BAK, B 106/9786/2; Neuordnung des Fürsorgerechts, S.262f.; Wirtschafts- und Sozialpolitik. Informationsdienst für die Deutsche Wirtschaft 10 (1958), Nr. 46, S. 10; NDV 39 (1959), S. 132.

209 Vgl. Besprechung mit den Vertretern der obersten Landessozialbehörden am 21./22. 10. 1958, LAS Abt. 761 Nr. 8874; Stellungnahme des BMW vom 4.12.1958, BAK, B 106/9686; NDV 39 (1959), S. 132ff.; Lücke, Krankenhilfe. 
Krankenkassenleistungen bzw. nach Aussteuerung, beließ deren Ausgestaltung allerdings wie bisher im Ermessen des Sozialhilfeträgers. ${ }^{210}$

Verglichen mit dem ersten Referentenentwurf waren also bereits im zweiten Entwurf vom März 1959 die gesundheitlichen Hilfen deutlich zurückgefahren worden und blieben inhaltlich auch im wesentlichen unverändert bis zur Verabschiedung des Gesetzes. Die Bundesregierung schätzte den materiellen Mehraufwand für dieses Gebiet auf zusammen knapp 40 Mio. DM; bei einem Aufwand von rund 250 Mio. DM im Jahre 1958 entsprach dies einer Kostensteigerung um $20 \%$. Den - auch im Vergleich zu den übrigen Hilfen in besonderen Lebenslagen - größten Anteil mit gut 33 Mio. DM stellte die Krankenhilfe, obwohl das materielle Leistungsrecht hier nicht wesentlich geändert worden war. Kostensteigernd - und damit für eine politische Intervention interessant - waren hier also nicht die Leistungen selbst, sondern die erweiterten Einkommensgrenzen, die den Eigenbeitrag der Leistungsempfänger senkten und die potentielle Klientel erweiterten. ${ }^{211}$

Im Bundestagsplenum machte sich dann die FDP im Interesse der niedergelassenen Ärzte gegenüber Polikliniken und Amtsärzten für die ausdrückliche Garantie des Rechtes der freien Arztwahl stark ${ }^{212}$, was die anderen Fraktionen zwar für überflüssig hielten, aber aus wahltaktischen Gründen nicht ablehnten. ${ }^{213}$ Die wachsenden Einflußmöglichkeiten der organisierten Ärzteschaft ${ }^{214}$ und der Trend zur allmählichen Ausschaltung des öffentlichen Gesundheitsdienstes machten also auch vor der kommunalen Sozialhilfe nicht halt. Statt als Schrittmacher für eine umfassende Gesundheitsreform zu fungieren, hatte sich die Sozialhilfe in das bestehende Gesundheitssystem der Bundesrepublik eingepaßt. ${ }^{215}$

\section{Tuberkulosebilfe}

Hatte sich schon die Einbeziehung vorbeugender Gesundheitshilfen in das BSHG als schwierig erwiesen, so galt dies erst recht für die Tuberkulosehilfe. Denn hier spielten seuchenpolizeiliche Belange eine wichtige Rolle, die Hilfe war seit dem

210 Vgl. $₫ 37$ BSHG.

211 Vgl. die Begründung zum BSHG-Regierungsentwurf vom Februar 1960, S.66, BT, 3. Wp. 1957, Anlagen, Bd.67, Drs. 1799; Willi Bangert, Bundessozialhilfegesetz, S. 349.

212 Vgl. entsprechende Eingaben der Bundesärztekammer und der Kassenärztlichen Bundesvereinigung vom 4.10.1960, PA, Gesetzesmaterialien III/349 A 3, der Arbeitsgemeinschaft fachärztlicher Berufsverbände vom 13.1.1961, die in polemischer Form auch als Broschüre veröffentlicht wurde, ebenda, A 4 bzw. B, sowie zwei Schreiben des Verbands der Ärzte Deutschlands (Hartmannbund) an den Sozialpolitischen Arbeitskreis der CDU/CSU-Fraktion vom 21.2.1961 bzw. den Fraktionsvorsitzenden Krone vom 27.2.1961, ACDP, CDU/CSU-Fraktion AK IV, VIII-005-106/1; Änderungsantrag der FDP-Fraktion vom 2.5.1961, Umdruck 888 zur Bundestagssitzung am 4.5.1961, BT, 3. Wp. 1957, Sten. Ber., Bd. 49, S. 9192; Thieding, Bundessozialhilfegesetz.

213 Vgl. Sitzung des Bundestages am 4. 5. 1961, S. 9073, BT, 3. Wp. 1957, Sten. Ber., Bd. 49.

214 Vgl. Süß, Gesundheitspolitik, S. 93.

215 Zur jüngsten Neufassung der gesundheitlichen Hilfen in der Sozialhilfe durch das „Gesetz zur Modernisierung der gesetzlichen Krankenversicherung“ vom 14.11.2003, BGBl. I S. 2190, vgl. Schellhorn, Einordnung, S. 172. 
Krieg außerhalb des Fürsorgerechts geregelt und die Rentenversicherung, nicht die Fürsorgeverbände, waren ihre Hauptträger.

Solange Antibiotika noch nicht entwickelt bzw. der breiten Bevölkerung zugänglich waren, in der Bundesrepublik bis in die fünfziger Jahre, bildete die soziale Prophylaxe durch Gesundheitsfürsorge das entscheidende Mittel zur Bekämpfung dieser Volkskrankheit: von der Früherkennung über die Isolation des Kranken, Hygieneerziehung, Ernährungszulagen und Kuren bis hin zur Beschaffung einer neuen Arbeitsstelle. Zu einem planmäßigen Ausbau dieser Maßnahmen war es in den zwanziger Jahren in Deutschland nicht gekommen, vielmehr blieb die Tbc-Fürsorge nach dem finanziell bedingten Scheitern eines Reichstuberkulosegesetzes kommunaler und privater Initiative überlassen. 1928 bestanden immerhin gut 1400 professionell arbeitende Tuberkulosefürsorgestellen zur Ermittlung und Betreuung der Erkrankten und zur prophylaktischen Arbeit. ${ }^{216}$

Während des Nationalsozialismus wurden diese Maßnahmen durch Einschaltung der Gesundheitsämter zwar reichsweit weiter ausgebaut und vereinheitlicht und überdies die Möglichkeit der Zwangsasylierung Tuberkulöser eingeführt ${ }^{217}$, doch bei der Durchführung der eigentlichen Heilbehandlung bestand ein Wirrwarr der Kostenträgerschaft, was eine planmäßige Tuberkulosebekämpfung kaum zuließ.218 Da die Tuberkulose aber letztlich die Wehrfähigkeit der deutschen Soldaten bedrohte und überdies die NSV mit den staatlichen Maßnahmen konkurrierte, wurde auf Betreiben des „Reichsgesundheitsführers“ Conti am 8. September 1942 die „Verordnung über Tuberkulosehilfe“219 erlassen, die zum ersten Mal die einheitliche Behandlung von nicht versicherten Tbc-Kranken ermöglichte: indem generell die Gau(Landes-)fürsorgeverbände die Tuberkulosehilfe durchzuführen und zu finanzieren hatten und damit leistungsfähige Kostenträger geschaffen wurden; neben Heilbehandlung, Absonderung und Pflege für den Erkrankten und seine Familie auch wirtschaftliche Hilfe zu gewähren war, die über den Fürsorge-Richtsätzen lag; für die Hilfe eine Einkommensgrenze von 7200 RM im Jahr für Alleinstehende vorgesehen und damit der Empfängerkreis gegenüber der öffentlichen Fürsorge beträchtlich erweitert wurde; die Hilfe explizit nicht als Leistung der öffentlichen Fürsorge firmierte und damit die Rückerstattungspflicht entfiel. Diese Verordnung brachte die Träger der Sozialversicherung in Zugzwang, so daß diese im Sommer 1943 mit der Gründung eines „Tuberkulose-Versorgungswerks“ ihre Leistungen denen der Fürsorgeverbände anglichen. 1943/44 trug das Versorgungswerk rund vier Fünftel aller Tbc-Fälle, das restliche Fünftel trugen die Landesfürsorgeverbände. ${ }^{220}$ Den kriegsbedingten Mangel an dringend notwendigen Plätzen in Heilstätten und die gesundheitlichen Folgen der immer

216 Vgl. Sachße/Tennstedt, Geschichte, Bd. 2, S. $126 \mathrm{ff}$.

217 Was das für die Betroffenen bedeuten konnte, schildert eindrücklich Ayaß, „Asoziale“, S. 102ff., am Beispiel der Thüringer Landesheilanstalten in Stadtroda; dort mußten die Tuberkulosekranken unter gefängnisähnlichen Bedingungen leben und arbeiten und wurde teilweise gezielt Tabak verabreicht.

218 Vgl. Hansen, Wohlfahrtspolitik, S.291ff.; Sachße/Tennstedt, Geschichte, Bd. 3, S. 169ff.; NDV 30 (1950), S. $18 f$.

219 RGBl. I S. 549.

220 Vgl. Rundschreiben 1 (1946), S. 42. 
schlechteren Ernährungs- und Wohnsituation konnten freilich auch diese rechtlichen Verbesserungen nicht mehr auffangen.

Der alarmierende Anstieg der Tuberkulose in der Nachkriegszeit bei zunächst hoher Mortalitätsrate ${ }^{221}$ verlangte eigentlich nach einer Fortsetzung der 1942/43 viel zu spät rechtlich begründeten Maßnahmen. Tatsächlich wurde die Tuberkulose-Verordnung in den Ländern der Westzonen auch weiter angewendet, dabei allerdings durch Herabsetzung der Einkommensgrenze teilweise erheblich eingeschränkt und die wirtschaftliche Hilfe selbst ebenfalls vermindert. ${ }^{222}$ Denn die finanziell stark angeschlagenen Landesversicherungsanstalten stellten jetzt einen Teil der ihrer Ansicht nach 1943 nur freiwillig übernommenen Leistungen wieder ein, so daß hier die Fürsorgeverbände einspringen mußten und das alte Kostengerangel erneut begann. ${ }^{223}$ Geklärt wurde die Lage wieder einmal zuerst für die Empfänger von Kriegsfolgenhilfe, indem der Bund seit 1949/50 auch aufgrund der Tuberkulose-Verordnung geleistete Hilfen der Landesfürsorgeverbände zum Großteil finanzierte. ${ }^{224}$ Die Verhandlungen zwischen Rentenversicherungsträgern und Fürsorgeverbänden dagegen zogen sich über Jahre erfolglos hin: Die Rechtsverbindlichkeit der Regelungen von 1942/43 und folgender Ministerialerlasse war dabei ebenso umstritten, wie die Frage, inwieweit die Unterbringung der sogenannten Asylierungsfälle und die wirtschaftliche Betreuung der Familien von den Versicherten oder aber als Staatsaufgabe aus öffentlichen Mitteln zu finanzieren sei. ${ }^{225}$

Seit Anfang der fünfziger Jahre ging zwar die Zahl der tuberkulosebedingten Todesfälle deutlich zurück, doch die Rückfallquote blieb bedenklich hoch, da viele nicht mehr ansteckend Tuberkulöse wegen der geringen wirtschaftlichen Hilfen zu früh zu arbeiten begannen. ${ }^{226}$ Außerdem wurden rechtlich abgesicherte Rehabilitationshilfen für teilinvalide Tbc-Kranke immer dringender. Rechtsunsicherheit sowie uneinheitliche und ungenügende Leistungen begründeten daher die Forderung nach einem Bundesgesetz über Tuberkulosehilfe noch vor einer allgemeinen sozialen Neuordnung. ${ }^{227}$ Schließlich forderte auch der Bundestag einstimmig auf Antrag der SPD-Fraktion am 24. März 1955 die Bundesregierung zur Vorlage eines „seit langer Zeit angekündigte[n]“ Gesetzes über Tuberkulosehilfe

221 Berlin hielt einen traurigen Rekord mit einer Sterblichkeitsziffer 1945 von 29,1 auf 10000 Einwohner gegenüber 8,2 im Jahre 1938; vgl. NDV 28 (1948), S. 134; auch Jochheim u.a., Rehabilitation, S. 573f., $582 \mathrm{f}$.

222 Vgl. NDV 27 (1947), S. 134ff.; 28 (1948), S. $32 \mathrm{ff}$.

223 Vgl. NDV 30 (1950), S. 18ff., $145 f$.

224 Vgl. \10,3 des Ersten Überleitungsgesetzes vom 28.11.1950, BGBl. S. 773.

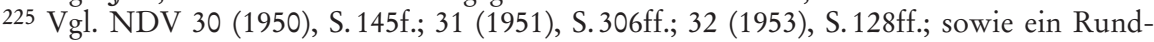
schreiben des BMI über Durchführung der VO über Tuberkulosehilfe vom 10.4.1954, GMBl. S. 197; ferner Schewe, Bedeutung, S. 147.

226 So sank zwar die Zahl der Todesfälle von 5 je 10000 Einwohner im Jahre 1949 auf 1,9 im Jahre 1956. Doch 1956 gab es in der Bundesrepublik und Berlin immer noch rund 443000 Tuberkulosekranke. Eine Stichprobe aus dem Jahr 1954 ergab, daß von rund 800 stationär behandelten Tbc-Kranken rund drei Viertel innerhalb von drei Jahren rückfällig geworden waren; vgl. Begründung zum Entwurf eines Gesetzes über die Tuberkulosehilfe 1958, S. 11f., BT, 3. Wp. 1957, Anlagen, Bd. 57, Drs. 349.

227 Vgl. NDV 32 (1952), S. $128 \mathrm{ff}$. 
auf. 228 Doch erst ein Jahr später, im März 1956, legte das federführende Bundesinnenministerium einen in jahrelangen Vorarbeiten entstandenen Gesetzentwurf vor. ${ }^{229}$ Schuld an der Verzögerung war in erster Linie die Weigerung der Rentenversicherungsträger, einen Rechtsanspruch auf das Heilverfahren zu gewähren ${ }^{230}$; außerdem gab es bei den Landkreisen Vorbehalte gegen ein möglicherweise großzügige Maßstäbe setzendes Sondergesetz vor einer allgemeinen Fürsorgerechtsreform. ${ }^{231}$ Umstritten zwischen Bundestagsmehrheit und Bundesrat war vor allem die Aufgabe der Rentenversicherung: Während Bundesrat und Vermittlungsausschuß einen gesetzlichen Rechtsanspruch auf Tuberkulosehilfe in der Rentenversicherung forderten, lehnten der Verband der Rentenversicherungsträger und schließlich die Regierungsmehrheit im Bundestag dies als Eingriff in die Selbstverwaltungsrechte der Sozialversicherung ab; mit der Ablehnung des Entwurfs auch durch den Bundesrat am 6. September 1957 war das Gesetz kurz vor Ablauf der zweiten Wahlperiode endgültig gescheitert. ${ }^{232}$

Die Bundesregierung legte dem Dritten Bundestag dann einen neuen Gesetzentwurf vor, der im wesentlichen dem Vermittlungsvorschlag folgte und vom Bundestagsausschuß für Kommunalpolitik und öffentliche Fürsorge im November 1958 kaum geändert verabschiedet wurde. ${ }^{233} \mathrm{Da}$ jedoch die Rentenversicherungsträger abermals gegen den geplanten Rechtsanspruch opponierten und die Länder eine stärkere Kostenbeteiligung des Bundes forderten, wurde die zweite und dritte Lesung um drei Monate verschoben; nach schwierigen Verhandlungen stimmte der Bundestag schließlich auf einen interfraktionellen Antrag hin einem prinzipiell weitgehenden, in den Einzelheiten aber stark eingeschränkten Rechtsanspruch im Rahmen der Rentenversicherung am 18. März 1959 zu. ${ }^{234}$ Nach abermaliger Anrufung des Vermittlungsausschusses wurde das Gesetz schließlich am

228 Antrag der SPD-Fraktion betr. Tuberkulosehilfe vom 16.2.1955, BT, 2. Wp. 1953, Anlagen, Bd. 34, Drs. 1208; Bundestagssitzung am 24.3.1955, BT, 2. Wp. 1953, Sten. Ber., Bd. 24, S. $4198 \mathrm{f}$.

229 Entwurf eines Gesetzes über die Tuberkulosehilfe (THG) 1956, BT, 2. Wp. 1953, Anlagen, Bd. 41, Drs. 2213.

230 Vgl. Hagen, Gesundheitsfürsorge, S. 244.

231 Vgl. entsprechende Schreiben des DLT an den Bundesinnenminister vom 30.8.1954 sowie an den Bundesratsausschuß für Innere Angelegenheiten vom 13.6.1955, BAK, B 172/444-01/1.

232 Vgl. die knappe Darstellung bei Schewe, Bedeutung, S.147f.; ferner das Vorwort zum THG-Kommentar von Muthesius u.a., Recht, S. IXff.; ferner den Schriftlichen Bericht des Bundestagsausschusses für Fragen der öffentlichen Fürsorge vom 6.5.1957, BT, 2. Wp. 1953, Anlagen, Bd.52, Drs. 3489 sowie zu 3489 (sic!); Bundestagssitzung am 26.6.1957, S. 12682ff., BT, 2. Wp. 1953, Sten. Ber., Bd.37; Verlangen des Bundesrates auf Einberufung des Vermittlungsausschusses vom 12.7.1957, BT, 2. Wp. 1953, Anlagen Bd.54, Drs. 3735; Mündlicher Bericht des Vermittlungsausschusses vom 25.7.1957, ebenda, Drs. 3751; Sitzung des Bundesrates am 6. 9.1957, BR 1957, Sten. Ber., S. $780 f$.

233 Vgl. Entwurf eines Gesetzes über die Tuberkulosehilfe (THG) 1958, BT, 3. Wp. 1957, Anlagen, Bd.57, Drs. 349; Schriftlicher Bericht des Ausschusses für Kommunalpolitik und öffentliche Fürsorge vom 26.11.1958, BT, 3. Wp. 1957, Anlagen, Bd.59, Drs. 680; NDV 38 (1958), S. $149 \mathrm{ff}$.

234 Vgl. die Sitzungen des Bundestages am 11.12.1958, S. 2909, BT, 3. Wp. 1957, Sten. Ber., Bd. 42, sowie am 18.3.1959, S.3539, ebenda, Bd. 43. 
3. Juni 1959 vom Bundestag verabschiedet ${ }^{235}$ und konnte nach Zustimmung des Bundesrates am 1. Oktober 1959 in Kraft treten. ${ }^{236}$

Die entscheidende Neuerung des Gesetzes betraf die Rentenversicherung: Tuberkulosekranke Versicherte (ähnlich ihre Ehegatten und Kinder) und Rentner hatten künftig einen Rechtsanspruch auf Maßnahmen zur Wiederherstellung der Erwerbsfähigkeit (Heilbehandlung, Berufsförderung und soziale Betreuung). Damit war endlich eine klare Abgrenzung zwischen den beiden wichtigsten Trägern der Tuberkulosehilfe, zwischen Rentenversicherung und Fürsorgeverbänden möglich, die überdies den realen Verhältnissen entsprach: 1955 hatten die Rentenversicherungen rund 251 Mio. DM, die Landesfürsorgeverbände etwa 110 Mio. DM für die Tuberkulosehilfe aufgewendet. ${ }^{237}$ Ausdrücklich schrieb das Gesetz die Zusammenarbeit der verschiedenen Träger der Tbc-Hilfe vor und wies dabei den Gesundheitsämtern als zentraler Antrags- und Koordinierungsinstanz eine wichtige Aufgabe zu.

Für die Fürsorge war die wesentliche Neuerung die Statuierung eines Rechtsanspruchs auf Tbc-Hilfe im Rahmen einer begrenzten Nachrangigkeit ${ }^{238}$, nachdem das Bundesverwaltungsgericht noch 1955 einen solchen Anspruch verneint hatte. ${ }^{239}$ Auf diese Weise war im Rahmen des traditionellen vielgliedrigen Sozialleistungssystems die seuchenhygienisch gebotene Mindestversorgung eines jeden Tuberkulösen gesichert. Diese Hilfe umfaßte nun erstmals neben der Heilbehandlung und der wirtschaftlichen Hilfe auch Eingliederungshilfe und vorbeugende Hilfe. Art und Maß der Leistungen lagen im pflichtmäßigen Ermessen des jeweiligen Leistungsträgers. Auch weiterhin war eine Rückerstattung der Leistungen ausgeschlossen. Mit einem Freibetrag von 660 DM an monatlichem Einkommen für ein Ehepaar ohne Kinder sicherte das Gesetz wieder Hilfsmaßnahmen auch für den unteren Mittelstand. Oberhalb dieser Grenze konnte ein Kostenbeitrag verlangt werden. Kaum für das gesamte BSHG-Projekt als Beispiel geplant, sondern vor allem den neuen Leistungspflichten der Rentenversicherung wie dem allgemeinen Seuchenschutz geschuldet, war die erhebliche Beteiligung des Bundes an der Finanzierung der Hilfen: Bei der Rentenversicherung trug er die Kosten

235 Vgl. Mündlicher Bericht des Vermittlungsausschusses vom 24.4.1959, BT, 3. Wp. 1957, Anlagen, Bd.61, Drs. 1034; Sitzung des Bundestages am 3.6.1960, S.3677ff., BT, 3. Wp. 1957, Sten. Ber., Bd. 43.

236 „Gesetz über die Tuberkulosehilfe“ (THG) vom 23.7.1959, BGBl. I S. 513. Zur versicherungsrechtlichen Bedeutung (die Rentenreform hatte allgemeine Rehabilitationsmaßnahmen als Kann-Leistung belassen) vgl. Schewe, Bedeutung, S.148. Zum THG insgesamt die ausführliche Erläuterung von Spahn, Zum Tuberkulosehilfegesetz; ferner Bogs, Gesetzgebung, S. 271f.

237 Vgl. Schewe, Bedeutung, S. 148.

$238 \int 1$ THG regelte im Interesse der Seuchenbekämpfung die Subsidiarität der Fürsorge insofern eigenständig und anders als von den kommunalen Trägern für das BSHG gewünscht, als die LFV nur gegenüber Pflicht-, nicht aber gegenüber Kann-Leistungen anderer Träger subsidiär waren; hier griff das Nachrangprinzip nur dann, wenn auch die Kann-Leistung tatsächlich gewährt wurde; vgl. Spahn, Zum Tuberkulosehilfegesetz, S. 326 .

239 Entscheidungen vom 15.9.1955, Fürsorgerechtliche Entscheidungen der Verwaltungsgerichte Bd. II, Nr. 1 und 2; vgl. Jehle, Fürsorgerecht, S. 493. 
für die stationäre Dauerbehandlung, bei der Fürsorge die Hälfte davon sowie die Hälfte der Eingliederungshilfe, der besonderen wirtschaftlichen und der vorbeugenden Hilfe und verschiedener Beihilfen.

Das THG knüpfte also an die Verordnung von 1942 an, war aber nach Diktion und erweitertem Umfang der Hilfe, wie Dieter Schewe 1959 treffend feststellte, „wohl am richtigsten als ein Vorläufer des neuen Bundessozialhilfegesetzes“ anzusehen. ${ }^{240}$ Tatsächlich war die weitestgehend unveränderte Einarbeitung dieses Sondergesetzes in das BSHG von der Sozialabteilung auch von Anfang an geplant $^{241}$ : Damit kam die Sozialabteilung einerseits den kommunalen Trägern entgegen, indem künftigen Wünschen nach Sonderbehandlung auch anderer Gruppen von vornherein ein Riegel vorgeschoben und die Verwaltungspraxis vereinfacht wurde. Andererseits bildeten KBG und THG für Scheffler und die Fürsorgereformer um Muthesius gleichsam das Einfallstor in das BSHG für höhere und neue Leistungsstandards, konnte man doch hinter einmal Gesetz gewordene Rechtsansprüche oder Einkommensgrenzen nicht mehr zurückgehen. ${ }^{242}$

Gegner dieser Inkorporation waren zum einen der Reichsbund, zum anderen die Gesundheitsabteilung des Bundesinnenministeriums wie auch die obersten Medizinalbeamten der Länder. Der Widerstand des Reichsbunds galt primär natürlich dem Einbau des KBG, er konnte aber einem Einbau des THG als Präzedens ebenfalls nicht das Wort reden. ${ }^{243}$ Die Gesundheitsabteilung hingegen lehnte wegen der Pläne für ein Bundesgesundheitsgesetz die Einfügung Anfang 1958 entschieden ab: Allgemeine gesundheitsrechtliche Vorschriften über seuchenhygienische Maßnahmen und Zuständigkeiten gehörten nicht in ein Fürsorgegesetz. ${ }^{244}$ Hier stellte man sich angesichts der schwierigen Genese des THG wie des KBG die Frage, ob es denn zweckmäßig sei, diese abermals der parlamentarischen Behandlung auszusetzen. ${ }^{245}$ Gottschick blieb demgegenüber unnachgiebig, räumte aber ein, daß die allgemeinen gesundheitsrechtlichen Vorschriften im BSHG nur übergangsweise bis zur Verabschiedung eines allgemeinen Gesundheitsgesetzes aufgeführt werden sollten, um eine Gesetzeslücke zu vermeiden.

Nachdem Ende Juli 1959 das THG endlich verabschiedet war, kam es über Form und Umfang seiner Aufnahme in das künftige BSHG allerdings zu Streitigkeiten zwischen den beteiligten Bundesressorts, was die Vorlage im Kabinett weiter verzögerte. Uneinigkeit herrschte darüber, wie die Bestimmungen über Tuber-

240 Schewe, Bedeutung, S. 148.

241 Vgl. Abschnitt 10 des BSHG-Entwurfs vom 24.11.1956, BAK, B 106/20648; Vermerk Referat V A 4 vom 29.6.1957, BAK, B 106/20652; Allgemeine Bemerkungen zum BSHG-Entwurf 7/1958, S. 28, BAK, B 106/20643.

242 Vgl. Gerhard Scheffler, Neuordnung, S. 26; NDV 38 (1958), S. 305, 309 f.

243 Vgl. Stellungnahme des Reichsbundes der Kriegs- und Zivilbeschädigten, Sozialrentner und Hinterbliebenen zum BSHG-Entwurf vom Juli 1958 [5.11.1958], BAK, B 106/9686.

244 Vgl., auch zum Folgenden, Vermerke Referat V A 4 vom 18.2. und 5.3.1958, BAK, B 106/20643; Dr. Karl [Äußerungen leitender Medizinalbeamter der Länder zum BSHGEntwurf, o.D.] mit handschriftlichem Vermerk Gottschicks vom 27.4.1959, BAK, B $106 / 20644$.

245 Vgl. Abteilung IV an Abteilung V am 11.6.1958, BAK, B 106/20643. 
kulosebekämpfung außerhalb der Sozialhilfe zu behandeln waren: Auf Wunsch des Bundesarbeitsministeriums, vom Finanzministerium unterstützt, plante die Sozialabteilung, diese Bestimmungen vorläufig als Restgesetz bestehen zu lassen, um die politischen Schwierigkeiten einer abermaligen Änderung des Versicherungs- und Versorgungsrechts zunächst zu vermeiden. ${ }^{246}$ Im Justizministerium hingegen beanstandete man den geplanten Torso aus gesetzestechnischen Gründen, hielt ihn für sinnentleert und forderte eben diese gleichzeitigen Gesetzesänderungen. Auf einer Staatssekretärsbesprechung am 21. Oktober 1959 einigten sich die beteiligten Ressorts schließlich auf einen Kompromiß: Das THG wurde als Ganzes aufgehoben und die Tuberkulosebekämpfung außerhalb der Sozialhilfe ohne inhaltliche Veränderungen als Übergangsbestimmung in das BSHG aufgenommen. ${ }^{247}$ Auch die übrigen Teile des THG wurden bis auf geringe Abweichungen in das Sozialhilfegesetz übernommen, wobei die Beteiligung des Bundes an den Kosten dieser Hilfen bestehen blieb. ${ }^{248}$ Der unproblematische Einbau war nur deshalb möglich geworden, da zentrale Innovationen des THG (und des KGB) wie der Rechtsanspruch, der Wegfall der Kostenersatzpflicht, der deutlich erweiterte Hilfsbedürftigkeits-Begriff, die vorbeugende und nachgehende Fürsorge sowie die verstärkte Rehabilitation Eingang in das allgemeine Fürsorgerecht gefunden hatten. Der Wunsch nach einem einheitlichen neuen Fürsorgerecht ohne Sondergesetze hatte sich damit auch als Schrittmacher für eine Verrechtlichung und Leistungsverbesserung eben dieses Rechts erwiesen. ${ }^{249}$

\section{Hilfe zur Pflege und zur Weiterführung des Haushalts}

Das Bestreben der Fürsorgereformer, den Schwerpunkt künftiger Sozialhilfetätigkeit auf die individuelle soziale Dienstleistung zu verlagern, illustriert besonders deutlich der geplante Ausbau der pflegerischen und häuslichen Hilfen. Über deren Notwendigkeit herrschte unter Fachleuten Mitte der fünfziger Jahre breiter Konsens: Die wachsende Zahl (dauerhaft) pflegebedürftiger alter Menschen, die Pflege Behinderter und alleinstehender Kranker oder die häusliche Nachsorge für

246 Vgl. Duntze an BMJ am 27.8.1959, BAK, B 106/20653; Abteilung V an Referat V A 2 am 17.9.1959, BAK, B 106/20647; Vermerk Bangert vom 23.9.1959, BAK, B 172/444$02 / 2$.

247 Vermerke Referat V 4 vom 22.10.1959, BAK, B 106/20647, vom 19.11. (über 16.11.) und 23.11.1959 (über 21.11.) über Besprechungen mit BMJ-Vertretern, BAK, B $106 / 20653$.

248 Vgl. SS 48ff. BSHG; Spahn, Vom Tuberkulosehilfegesetz zum Bundessozialhilfegesetz; NDV 41 (1961), S.337f.

2491970 betrug die Zahl der Empfänger von Tbc-Hilfe nach dem BSHG noch 80 000, 1983 nur noch 3 600, wobei 98\% der Kosten auf die Heilbehandlung entfielen. Wegen der rapide gesunkenen Bedeutung der Tuberkulose in der Bundesrepublik wurden die Bestimmungen über diese Hilfe im BSHG ab 1.1.1987 außer Kraft gesetzt, vgl. Art.26 des Zweiten Rechtsbereinigungsgesetzes vom 16.12.1986, BGBl. I S. 2441. Dementsprechend waren seitdem bei Tuberkuloseerkrankung oder -gefährdung die allgemeinen Bestimmungen des BSHG (vorbeugende Gesundheitshilfe, Krankenhilfe etc.) anzuwenden; vgl. Oestreicher/Schelter/Kunz/Decker, Bundessozialhilfegesetz, S. $17 f$. 